\title{
Designing a Multi-purpose GSM Based Interactive Embedded Data Acquisition System Providing Solutions for Fire Accidents
}

\author{
P. Michael Preetam Raj ${ }^{1}$, Rakesh Tirupathi ${ }^{1}$, P. Gopi Krishna ${ }^{2}$, Jasti Sateesh ${ }^{3}$, \\ Vijay Krishna Balachander ${ }^{4}$ \\ ${ }^{1,3}$ Department of ECE, KL University, Vijayawada, India \\ ${ }^{2}$ Department of ECM, KL University, Vijayawada, India \\ ${ }^{4}$ Senior Software Engineer, Bank of America, Hyderabad, India
}

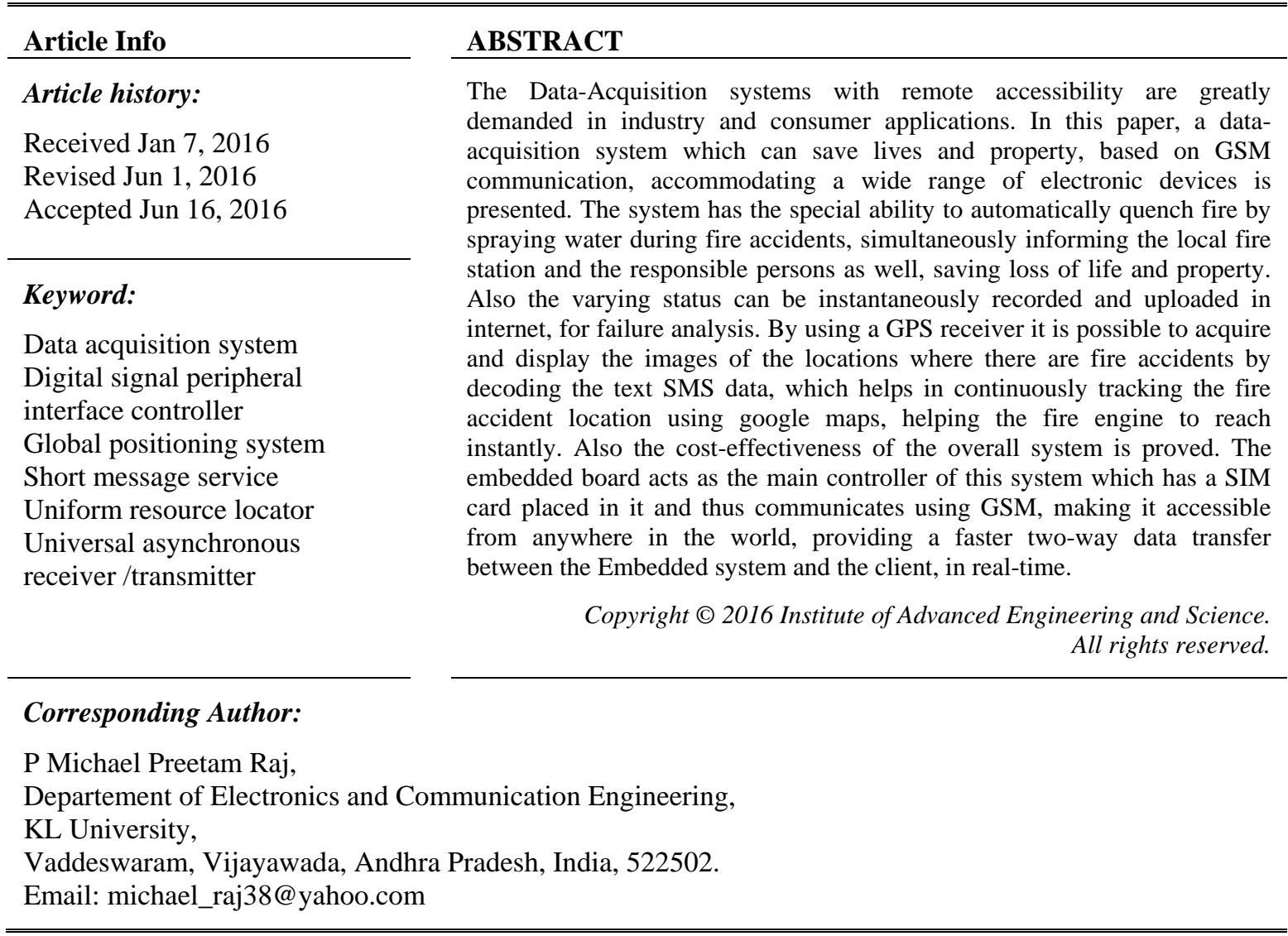

\section{INTRODUCTION}

Data acquisition systems are products and processes used to collect information to document or analyse some phenomenon which can be used at the client end for various applications as it is the process of sampling signals measuring real world physical conditions and converting the resulting samples into digital values that can be manipulated accordingly by a computer. A low-cost data acquisition and control system based on Internet has been designed and implemented in [1], where the images were acquired using a camera. A implementation of mobile communication technology to remote data transmission and control, including global positioning system, global system for mobile (GSM), and geographic information system was considered in [2]. A data transfer but unidirectional is presented in [3], which uses the GSM communications. A system which can achieve many tasks such as data acquisition of agricultural sensors, GPS information and navigation is presented in [4]. Solution for flight control and surveillance in real time for unmanned air vehicles is provided in [5]. Performance needs and constraints of a system and their solutions are discussed in [6]. Surface displacement measurements for 3 years obtained by space-borne synthetic aperture radar (SAR) observations over the Argentière glacier in the Mont-Blanc massif, France is presented in [7]. Solutions to the orientation of the tunnel using GPS and precise traversing are presented in [8]. 
The introduction of the tide data acquisition and processing for GPS buoys that can measure water surface elevations using real-time kinematic GPS technology is provided in [9]. The use of GPS buoy observations for precise determination of the tsunami source along with a tsunami warning system is presented in [10]. In this paper, a multi-purpose data-acquisition system based on GSM communication is presented. The embedded device sends the data whenever there is a sudden change in temperature or if there is a fire accident. Also it sends its data when it is requested by the client through an SMS. Thus the two-way transfer of data is presented. This proposed system eliminates the need for manual intervention and thus reduces the reaction time of response which is a huge benefit during emergency. Also it transmits the images in a much reduced data size as the transmission of the text data, containing the image data, is done through the GSM communication. Internet is required on the client side to obtain the image from the image data.

The design of the system can be such that any different sets of electronic devices can be interfaced onto the embedded board. However it requires the modification of the corresponding part of the program controlling the overall functioning. In Section III an application of this system proving its efficiency using devices interfaced in the embedded board is provided. In this paper, a Global Positioning System (GPS) receiver, a power sensor, a fire sensor, an electric motor and a temperature sensor are interfaced in the embedded board as sample devices to demonstrate its efficiency. The practical applications of the overall system can be for saving from much damage of life and property in the case of fire accidents. Also it can be considered in a wide range of fields where frequent monitoring of temperature, etc. measurements are required. This system can be used for multipurpose data acquisition such as power measurement etc., as several of the sample sensors can be connected to the embedded board.

\section{GENERAL SYSTEM ARCHITECTURE}

A system monitoring a variety of data in real-time and providing the states of a laboratory, which can also communicate with PC, are proposed in [11]. A system which gathers the data at the target with a sensor network triggered by time, transmitting the measured values to a target server is proposed in [12].

The solution for software and hardware design of a web based embedded remote laboratory system monitoring environment is proposed in [13]. A system, with wireless sensors embedded in applications such as, Internet-capable devices and cameras generates an enormous volume of data bits is proposed in [14].

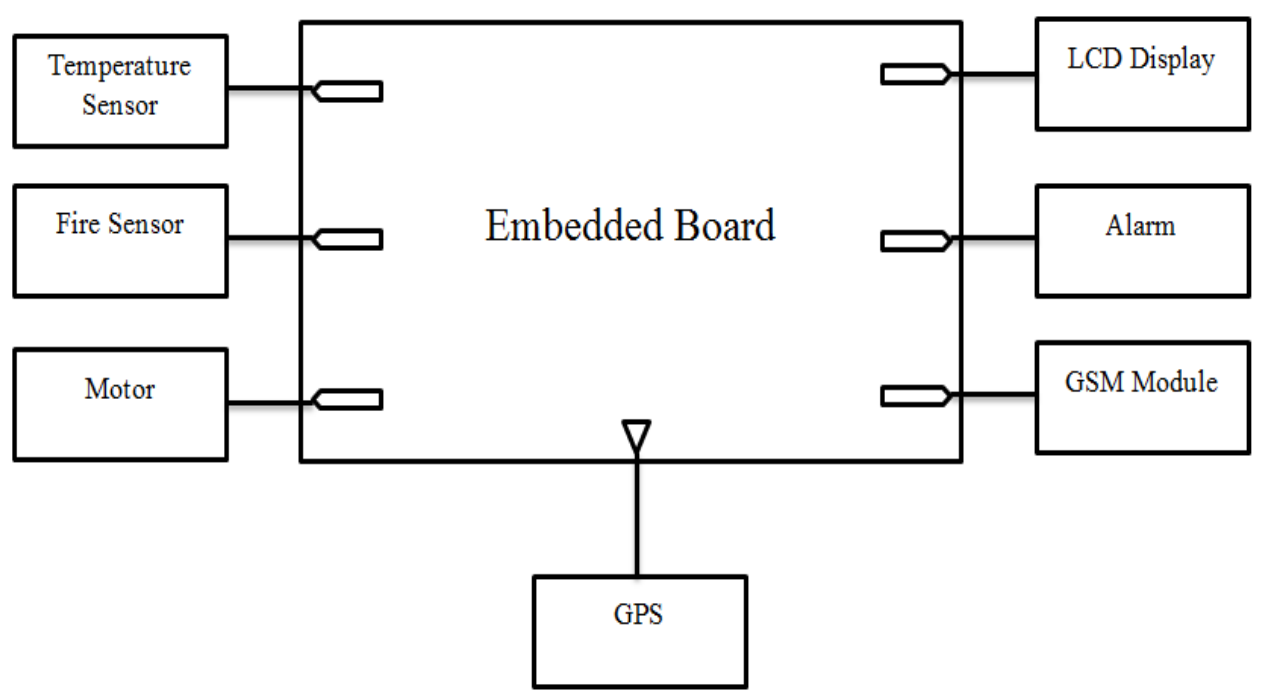

Figure 1. Block diagram of the embedded system with sample devices attached 


\section{PROPOSED SYSTEM ARCHITECTURE}

The proposed system architecture is shown in Figure 2.

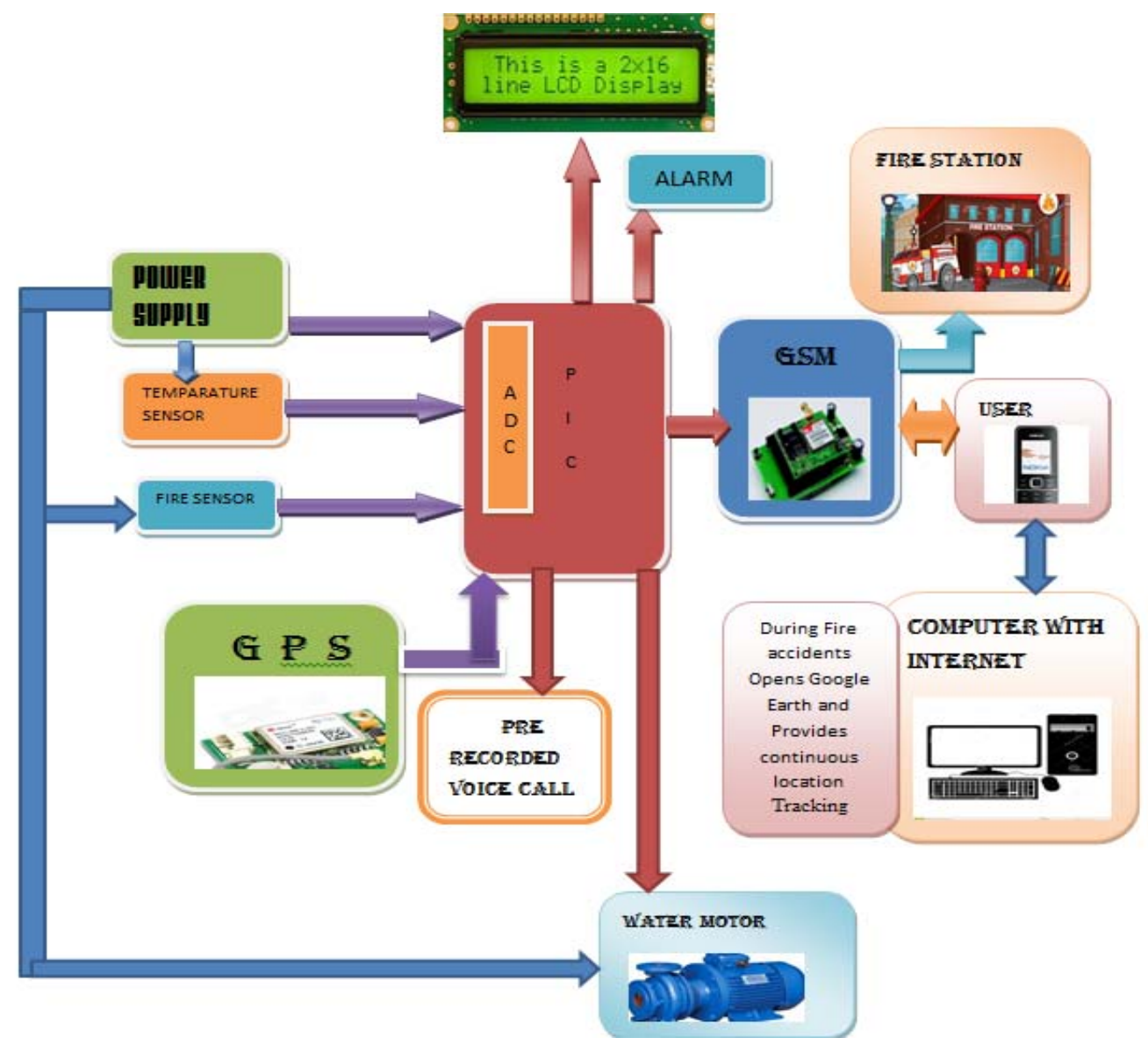

Figure 2. Block diagram of the embedded DAS with a set of attached devices

\section{DATA ACQUISITION SYSTEM BASED ON MOBILE COMMUNICATIONS}

Interactive embedded systems based on GSM communication can be designed to monitor and adjust using controlling of motor to automatically pump waters in the case of a fire accident. It is essential that the direction of the water pumping is so as to focus on areas which have a high probability of being sources of fire accidents. Multiple outlets can be provided from the same motor if the expected number of sources of fire accidents is large. In certain cases pumping of water to quench fire may not be suitable. In such cases an arrangement of sand containers above the expected areas of sources of fire accidents can be made available which have doors such that when opened the sand falls and automatically the fire is quenched.

This opening of the doors can be automatically controlled by running the motors, connected at the hinges of the doors, based on the data from micro-controller during fire accidents. Also data access is possible through messaging and a PC connected to internet, far away from the location. If the proposed system in this paper was not used, and image sending of the location of fire accident was necessary, then this should be implemented through a server, where the transmission of image is done by the internet leads, to poor data access speeds and costs. Also the major quality of providing safety is minimized in that case.

GSM communication was developed basically for cellular mobile communication. As mobile communication has become a necessity these days every individual is expected to have at least one SIM. Also, the GSM operators are providing GSM connection for free to increase the number of their customers. From the above two mentioned statements it is shown that the cost of obtaining a GSM connection can be nil. Also, cost effective schemes that mobile operators have come up with, especially in messaging where very high number of text messaging will cost very less are available. As the GSM communication is used for messaging, it is now proved that the overall cost of the communication in this system is very low. Further the minimization of costs can be done by having optimized data transfers using GSM. However this 
minimization of cost is negligible. The main challenge of the real time data transfer is that the data transfer rates should be within a favourable range. Since both the GSM communication and the internet which were used in this system completely isolated from each other in this system, operate at real time, this system is proved to be able operate at real time. The client can directly access the embedded DAS board if its contact number is made available to the client.

\subsection{Accessing the embedded board.}

There are several choices in obtaining data from the embedded board. One method is the embedded board sending its data to the client only when the client makes a request through an SMS. Second method is that the data can be messaged to the client at regular intervals. Also, any GSM subscriber who needs data can easily be given access by revealing the mobile number embedded in the DAS. Thus there are infinite ways of doing this. An optimized solution can be made based on the conveniences in the above cases. Also, most importantly, the data can be sent automatically to the client, fire station and other responsible personnel within few seconds when fire accident occurs. The Embedded system also automatically turns on the motors which pump water to quench fire which is an extremely efficient, simple and a low cost approach which acts a first-aid for fire accidents. In favourable case the fire may be completely quenched by the pumped water. In worst case, still there is no much problem as the water is pumped to minimize the adverse effects of fire and the board simultaneously and automatically calls for rescue. It will be further beneficial if the power supply in the area of fire accident is turned off, so that fire does not spread due to short-circuits. This can be done by automatically controlling a relay, which acts as a fuse connected to the main supply, based on the data from the micro-controller during fire accidents.

Thus, the safety measures provided by our system are endless and large in number, which can be implemented by making slight modifications to our proposed system. It is very much essential that the GSM signals are always reachable to the embedded device, the client, the other responsible and fire station personal. It is desired that the a specific time delay is provided after the enabling of the embedded device, as the GSM needs some time for start-up. It is a universally known fact that the GSM subscribers in this case the GSM modem in the embedded board and the clients including the fire station GSM subscribers operate at 900/1800 MHz frequencies. The transfer of messages are controlled by the AT commands. Also, the AT commands does the setup for GSM activities, which also includes checking the arrival of a new message, which provides specifications of the message format, information in the message, the destination identities to the embedded board and sending the recorded information to the clients who not only requested but also sends unrequested messages in case of fire accidents. Our system enables the clients to decode the message received and finds the location of fire accident and makes known the present condition from the decoded data. If the client is satisfied by the data in the text message it is not required to access internet which can also be done automatically by the corresponding program running in the micro-controller. This may lead in the reduction of a huge amount of data access time and cost. Also, when there is a requirement to know the location of the problem, which can be done by decoding the image, there is still only a very less access time required for accessing the data as there is only downloading from the web (google maps) without any initial upload.

Another important feature that this system provides is that the costs for data transfers can be decided by the client. If it is required a modification to the programming running in the embedded board can be used to transmit to a large number of clients. In such a case the contact numbers of those clients are definitely required to be specified to the embedded board. Another important feature of this system is that the transmission of bulky images is replaced by the corresponding transmission of their message containing the image data, which is a part of the SMS sent. There is a facility provided to the client at the receiver side that downloading of the image of the location of the fire accident can be done from google maps whose location was obtained from GPS receiver and transmitted to the client, from the above mentioned SMS data, by using a Computer system which is connected to internet using VB program, by making a specification of this image data as a part of URL, which returns the image that can be used to reach the spot easily as also the routes of travel are provided by the google maps. This has several advantages as not only the problem is reported but also the simultaneous action to reduce the fire effect by automatically pumping water by turning the motors on, and providing the paths of travel along with the simultaneous tracking of the vehicle can be done in two ways, i.e., by the google maps (global solution) and the GPS receiver (independent solution). Also data security is maintained as only those who know the phone number corresponding to the SIM card present in the embedded board can access the data. The data corresponding to the image is the latitude and longitude coordinates acquired and transmitted by the GPS receiver. Also, data security can further be enhanced by maintaining a password, to have secrecy of data, which should be revealed to those clients who want to access the system. The embedded device is programmed in $\mathrm{C}$ code and is debugged and downloaded into the embedded board using ICD-2. 


\subsection{Hardware specifications}

The heart of the embedded board used in this work is a dsPIC microcontroller. It has two UARTs internally available and has 48-KB of on-board erasable Flash memory. One UART is to communicate with the GSM modem. The other UART is to receive data from the GPS receiver. The Temperature sensor and fire sensor are interfaced to the analog pins after configuration. A mobile phone interfaced to PC with internet (or simply a mobile phone with internet facility) is used for the acquisition of the image at the receiving end.

\subsection{Software specification and its role}

The programming used in the microcontroller is done using C language. It conveys to the GSM modem that it has to send an SMS of the received data. The code running on the microcontroller initially also orders the embedded DAS to gather the data from all the sensors and GPS receiver interfaced onto the embedded board and messages it to the client when requested or sends automatically in case of fire accident, from the GSM modem. It also intimates the user if there are any errors in the connectivity. The further interfacing or manipulations to this system can simply be done by directly interfacing the devices onto the embedded board and making corresponding modifications to the code running in the microcontroller. Thus the system is very much flexible and can easily be modified based on various applications. The program can also be done such that it continuously sends the gathered data from the GPS, the temperature sensor and fire sensor, into the flash memory of the dsPIC such that the older data is replaced by the newer data.

\section{Nomenclatures}

\$GPGGA

\$GPBOD

Global positioning system fix data

Bearing origin to destination

\$GPGLL Geographic position of latitude or longitude

\$GPGSA GPS DOP and active satellites

\$GPGSV GPS Satellites in view

\$GPRMC Recommended minimum specific GPS or Transit data

\$GPVTG Track made and ground speed good

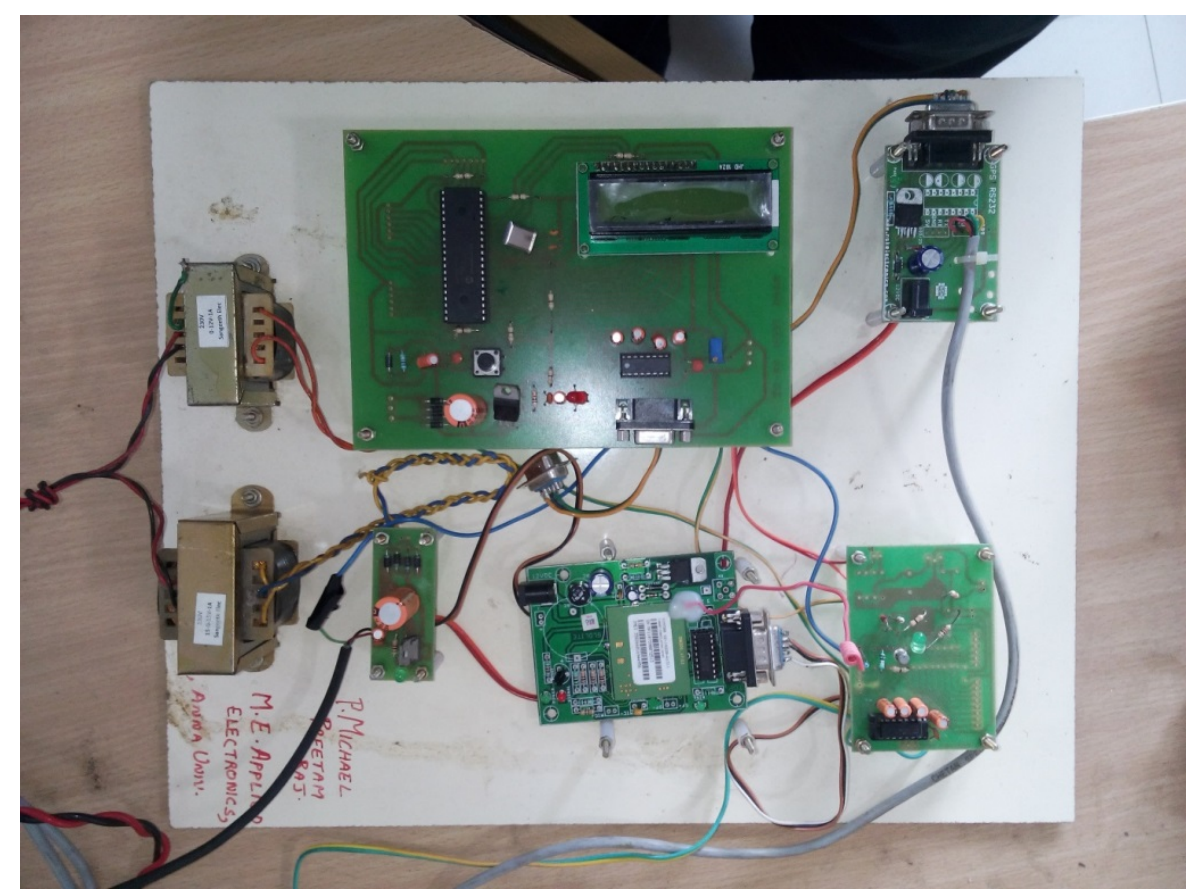

Figure 3. Photograph of the embedded DAS board and the mobile client with sample devices

\subsection{Example Application}

The data transfer in this system is as described below. It takes a time delay of 45s for the start-up of the SIM 300 GSM modem. For this example application, a temperature and fire sensors have been chosen. 
The data gathered from these sensors is converted into digital form and is forwarded to the micro controller. The program collects the data from the GPS receiver from a larger set of data and sends it to, the transmission register of the UART2 which is serially connected to the sr-92 GPS receiver. This is necessary as the GPS receiver provides several data, other than the latitude and longitude values, which are not necessary for this application. The required data can be gathered by comparing the starting characters of the strings shown in Figure 4.

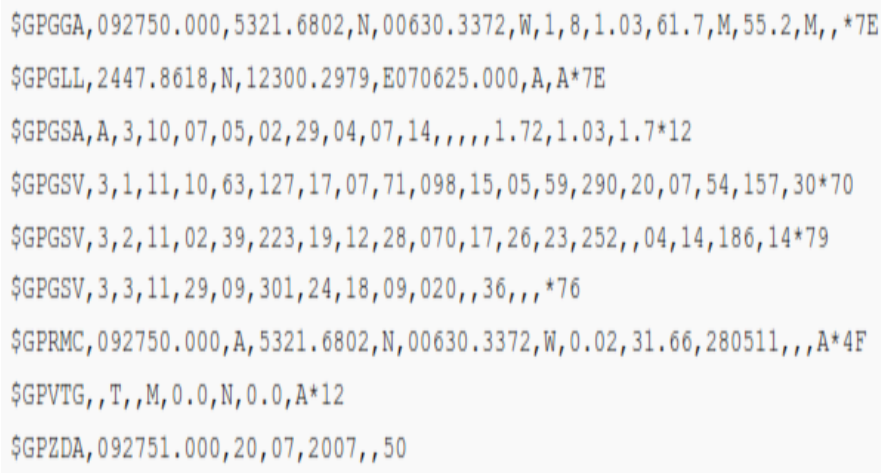

Figure 4. Single message sample

The example strings which contain the Latitude and longitude coordinates starts with "\$GPGGA". The coordinates of latitude and longitude values of a particular location are shown in Table 1. Thermistor is used as a temperature sensor to obtain the temperature measurement. The microcontroller has in-built analog to digital converter to which thermistor is interfaced.

The reduction of cost in this implemented system is to a great extent as the requirement of using a camera for obtaining images is eliminated. RS 232 is used in several interfacings such as to the GPS receiver, GSM modem and in-between the mobile phone and the PC. The approximate values of time shown in Table 2 not only prove the quickness of operation of this system but also further it can be maximized to a larger extent for the cases when high speed of data transfer is required. This high speed data transfers can be obtained by reducing the delay time provided in the $\mathrm{C}$ program in the microcontroller, as more than sufficient time is provided for the various devices to respond.

Table 1. Obtained latitude and longitude coordinates decoded from GPS receiver

\begin{tabular}{cc}
\hline Type & \$GPGGA \\
\hline Latitude & 16.4415 \\
North/South & $\mathrm{N}$ \\
Longitude & 80.6217 \\
East/West & $\mathrm{E}$ \\
\hline
\end{tabular}

Table 2. Conditions of FIRE detection and corresponding motor running status

\begin{tabular}{cccc}
\hline S. No & Fire detection & Motor Status & Approximate Time \\
\hline 1. & Fire available & ON (to pump water to quench fire) & 18 Second \\
2. & Fire not available & OFF & 17 Second \\
\hline
\end{tabular}




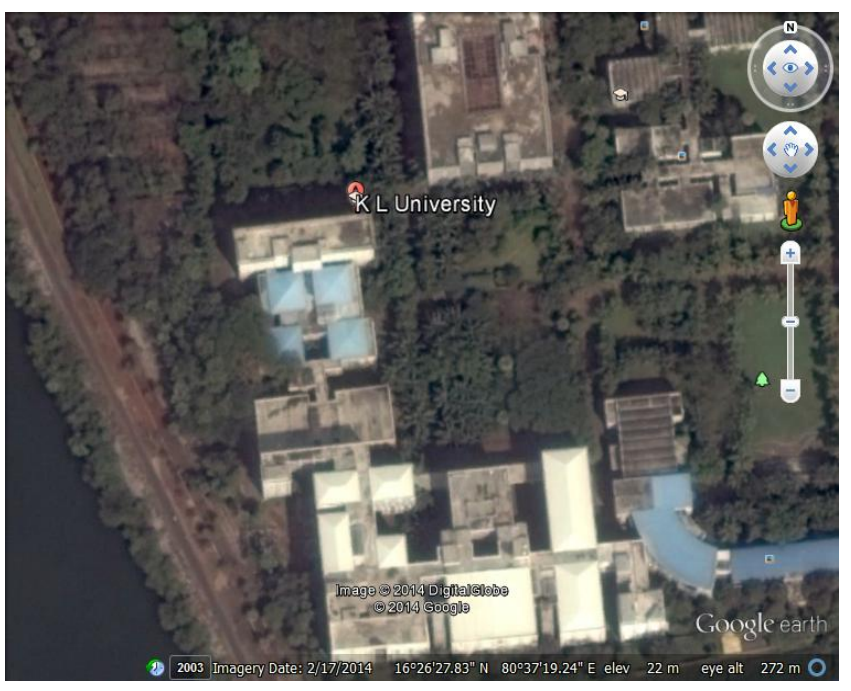

Figure 5. Expected Image corresponding to the above Table 1

\section{CONCLUSION}

In this work a data acquisition system which is proved to primarily provide safeties during fire accidents is provided. The main stunning feature of this system is that it automatically sends messages to the fire station personnel, owners of the system and anybody else who wants to be a part of it. Another fantastic feature of this system is that it also automatically turns the motors on to pump water to quench the fire. The third magnificent feature of this system is that it also automatically and spontaneously provides the location of the place where the fire accident occurs using google maps images which makes it very much easy for the fire station personnel and other related authorities to reach the place of problem within no time and thus minimize the advert effects of fire. The system also gives its users a wide range of options, in terms of the types of data, acquiring and accessing the data. An infinite number of applications can be implemented using this or a certain modification of this system. Thus there is this cool advantage of not having a necessity of using a camera for obtaining images of locations and uploading them into the internet, which further increases the data transfer rate, as images are directly obtained from google maps, and makes it preferable for all real-time and safety applications. As per the simplicity aspect of this system, use of bulky internet protocols is avoided and as the data is smartly compressed into an SMS. Thus it is understood this system provides a much needed improvements in terms of safety, costs, and simplicity and data access rates.

\section{ACKNOWLEDGEMENTS} Figure 5.

Our special thanks to google maps which is used to acquire the images of the locations shown in

\section{REFERENCES}

[1] A. Z. Alkar, et al., "An Internet-Based Interactive Embedded Data-Acquisition System for Real-Time Applications,” IEEE Transactions On Instrumentation and Measurement, vol/issue: 58(3), 2009.

[2] C. E. Lin, et al., "A real-time remote control architecture using mobile communication,” Dept. of Electr. Eng., Nat. Changhua Univ. of Educ., Taiwan, Instrumentation and Measurement, IEEE Transactions, 2003.

[3] E. Bekiroglu and N. Daldal, "Remote control of an ultrasonic motor by using a GSM mobile phone," Sens. Actuators a Phys., vol/issue: 120(2), pp. 536-542, 2005.

[4] Y. Dong and L. Wang, "Design of Embedded Cropland Data Acquisition and Processing System Based on Xscale and WinCE,” International Forum on Information Technology and Applications, vol/issue: 16(1), pp. 700 - 703, 2009.

[5] C. E. Lin and C. W. Hsu, "Verification of unmanned air vehicle flight control and surveillance using mobile communication,” Journal of Aerospace Computing, Information, and Communication, vol/issue: 1(4), pp. 189-197, 2004.

[6] S. Anvar and F. Louis, "The ANTARES offshore data acquisition: a highly distributed, embedded and COTS-based system,” Nuclear Science Symposium Conference Record, IEEE, vol/issue: 2(12), pp. 103-106, 2000. 
[7] F. Ponton and F. Vernier, "Observation of the Argentiere glacier flow variability from 2009 to 2011 by TerraSAR$\mathrm{X}$ and GPS displacement measurements," IEEE Journal of Selected Topics in Applied Earth Observations and Remote Sensing, vol/issue: 7(8), pp. 3274-3284, 2014.

[8] J. Zou and K. Tong, "Application of GPS control survey and precise traversing in the tunnel orientation,” Journal of Geomatics, vol/issue: 39(2), pp. 42-44, 2014

[9] H. Kawai and M. Satoh, "2010 Chile and 2011 Tohoku tsunami profiles measured by GPS buoys and coastal wave and tide gauges in a nationwide ocean wave information network for ports and harbors," Journal of Waterway, Port, Coastal and Ocean Engineering, vol/issue: 140(2), pp. 135-145, 2014.

[10] T. Kato and Y. Terada, "Tsunami due to the 2004 September 5th off the Kii peninsula earthquake, Japan, recorded by a new GPS buoy,” Journal of Earth, Planets and Space, vol/issue: 57(4), pp. 297-301, 2005.

[11] J. Linyiang, et al., "Embedded Laboratory Environment Monitor System," IEEE, WASE International Conference on Information Engineering, pp. 197-201, 2009.

[12] C. Tr"odhandl, et al., "Remote Target Monitoring in Embedded Systems Lab Courses using a Sensor Network,” pp. 5433-5438, 2006.

[13] V. Gupta, et al., "Sensor Network: An open data exchange for the web of things,” pp.753-755, 2010.

[14] W. Ping and W. Zheng, "IEEE, design and Implementation of open computer lab monitoring and management system,” IEEE, computer and modernization, vol. 11, pp. 125-128, 2007. 